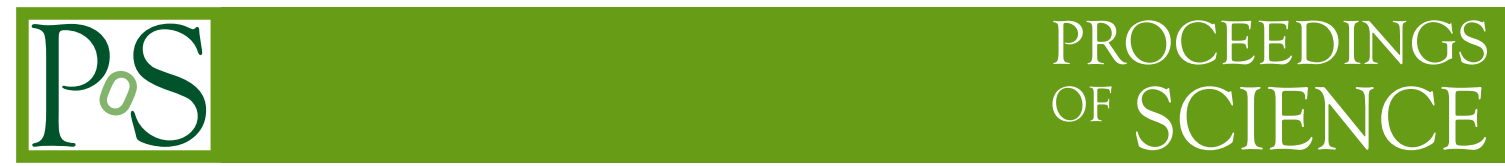

\title{
Perturbing QCD with external fields
}

\author{
Paolo Cea \\ Università di Bari, \& INFN - Bari, Italy \\ E-mail: paolo.cea@ba.infn.it
}

\section{Leonardo Cosmai*}

INFN - Bari, Italy

E-mail: leonardo.cosmai@ba.infn.it

\section{Massimo D'Elia}

Università di Genova \& INFN - Genova, Italy

E-mail: massimo.delia@ge.infn.it

We present some up-to-date results on QCD phase transition in a chromomagnetic constant background field and in an abelian monopole background field. Our results indicate that the QCD critical temperature is not modified by a monopole background field, whilst it is modified by a constant chromomagnetic field. We improve our earlier estimate of the QCD critical parameters in a chromomagnetic background field by performing lattice simulations with weaker fields.

The XXV International Symposium on Lattice Field Theory

July 30 - August 42007

Regensburg, Germany

${ }^{*}$ Speaker. 


\section{Introduction}

In previous studies $[1,2]$ on the vacuum dynamics of pure non-abelian gauge theories we found that the deconfinement temperature depends on the strength of an external abelian chromomagnetic field. We also verified that the same effect is not present in the case of abelian gauge theories, suggesting that it could be linked to the non-abelian nature of the gauge group. In our opinion the dependence of the deconfinement temperature on applied external fields is a consequence of the dynamics underlying color confinement and therefore, apart from possible phenomenological implications, such an effect could shed light on confinement/deconfinement mechanisms. On these premises it is important to test if the effect continues to hold and how it qualitatively changes when switching on fermionic degrees of freedom and on that ground to investigate the dependence of the deconfinement temperature on the strength of an external abelian chromomagnetic field in the case of full QCD with two flavors. Besides another relevant issue regards the relation between deconfinement and chiral symmetry restoration. As it is well known, the two phenomena appear to be coincident in ordinary QCD, while they are not so in different theories (like QCD with adjoint fermions [3-5]). Even if a simple explanation of this fact is still lacking it could be strictly connected to the very dynamics of color confinement. A contribution towards a clear understanding of this phenomenon could be to study whether it is stable against the variation of external parameters. In the present we investigated whether the deconfinement temperature depends on the strength of an external abelian chromomagnetic field and whether deconfinement and chiral symmetry restoration for QCD with two flavors coincide even in presence of a constant chromomagnetic field.

\section{A constant chromomagnetic field on the lattice}

QCD dynamics in presence of a time-independent background field at finite temperature can be studied [6-8] by means of the free energy functional

$$
\mathscr{F}\left[\vec{A}^{\mathrm{ext}}\right]=-\frac{1}{L_{t}} \ln \left\{\frac{\mathscr{Z}_{T}\left[\vec{A}^{\mathrm{ext}}\right]}{\mathscr{Z}_{T}[0]}\right\} .
$$

with $\mathscr{Z}_{T}$ the thermal partition functional

$$
\begin{aligned}
\mathscr{Z}_{T}\left[\vec{A}^{\mathrm{ext}}\right] & =\int_{U_{k}\left(L_{t}, \vec{x}\right)=U_{k}(0, \vec{x})=U_{k}^{\mathrm{ext}}(\vec{x})} \mathscr{D} U \mathscr{D} \psi \mathscr{D} \bar{\psi} e^{-\left(S_{W}+S_{F}\right)} \\
& =\int_{U_{k}\left(L_{t}, \vec{x}\right)=U_{k}(0, \vec{x})=U_{k}^{\mathrm{ext}}(\vec{x})} \mathscr{D} U e^{-S_{W}} \operatorname{det} M .
\end{aligned}
$$

The functional integration is performed over the lattice links, but constraining the spatial links belonging to a given time slice (say $x_{t}=0$ ) to be

$$
U_{k}\left(\vec{x}, x_{t}=0\right)=U_{k}^{\mathrm{ext}}(\vec{x}), \quad(k=1,2,3),
$$

$U_{k}^{\text {ext }}(\vec{x})$ being the elementary parallel transports corresponding to the external continuum gauge potential. In the previous equations $\vec{A}^{\mathrm{ext}}(\vec{x})$ is the continuum gauge potential of the external static background field, $S_{W}$ the standard pure gauge Wilson action, $S_{F}$ is the fermion action and $M$ is the fermionic matrix. The spatial links are constrained to the values corresponding to the lattice version 
of the external background field, whereas the fermionic fields are not constrained. In the case of a static background field which does not vanish at infinity we must also impose that, for each time slice $x_{t} \neq 0$, spatial links exiting from sites belonging to the spatial boundaries are fixed according to eq. (2.3). In the continuum this last condition amounts to the requirement that fluctuations over the background field vanish at infinity.

We compute by lattice simulations the derivative $F^{\prime}$ with respect to the inverse gauge coupling

$$
F^{\prime}(\beta)=\frac{\partial \mathscr{F}(\beta)}{\partial \beta}=\left\langle\sum_{x, \mu<v} \frac{1}{3} \operatorname{Re} \operatorname{Tr} U_{\mu v}(x)\right\rangle_{0}-\left\langle\sum_{x, \mu<v} \frac{1}{3} \operatorname{Re} \operatorname{Tr} U_{\mu v}(x)\right\rangle_{\vec{A}^{\mathrm{ext}}},
$$

where the subscripts on the averages indicate the value of the external field. Only unconstrained plaquette are taken into account in the sum in eq. (2.4).

In the present work we have considered a static constant abelian chromomagnetic field. The continuum gauge potential giving rise to a static constant abelian chromomagnetic field directed along spatial direction $\hat{3}$ and direction $\tilde{a}$ in the color space can be written in the following form:

$$
\vec{A}_{a}^{\mathrm{ext}}(\vec{x})=\vec{A}^{\mathrm{ext}}(\vec{x}) \delta_{a, \tilde{a}}, \quad A_{k}^{\mathrm{ext}}(\vec{x})=\delta_{k, 2} x_{1} H
$$

In SU(3) lattice gauge theory the constrained lattice links (see eq. (2.3)) corresponding to the continuum gauge potential eq. (2.5) are (choosing $\tilde{a}=3$, i.e. abelian chromomagnetic field along direction $\hat{3}$ in color space)

$$
\begin{aligned}
U_{1}^{\mathrm{ext}}(\vec{x}) & =U_{3}^{\mathrm{ext}}(\vec{x})=\mathbf{1}, \\
U_{2}^{\mathrm{ext}}(\vec{x}) & =\left[\begin{array}{ccc}
\exp \left(i \frac{a g H x_{1}}{2}\right) & 0 & 0 \\
0 & \exp \left(-i \frac{a g H x_{1}}{2}\right) & 0 \\
0 & 0 & 1
\end{array}\right] .
\end{aligned}
$$

Since our lattice has the topology of a torus, the magnetic field turns out to be quantized

$$
a^{2} \frac{g H}{2}=\frac{2 \pi}{L_{1}} n_{\mathrm{ext}}, \quad n_{\mathrm{ext}} \text { integer } .
$$

In the following $n_{\text {ext }}$ will be used to parameterize the external field strength.

\section{Deconfinement temperature and critical field strength}

Numerical simulations have been performed on $32^{3} \times 8$ and $64 \times 32^{2} \times 8$ lattices, using computer facilities at the INFN apeNEXT computing center in Rome. A slight modified version of the standard HMC R-algorithm [9] has been adopted to simulate QCD with two degenerate flavors of staggered fermions of mass $a m_{q}=0.075$. The critical gauge coupling has been determined from the position of the peak in $F^{\prime}\left[\vec{A}^{\text {ext }}\right]$ (eq. (2.4)), the derivative of the free energy with respect to the gauge coupling $\beta$, as a function of $\beta$. In figure 1 we show an example of $F^{\prime}$ measured for $n_{\mathrm{ext}}=1$ on a $32^{3} \times 8$ lattice. In the same figure we display also the chiral condensate $\langle\bar{\psi} \psi\rangle$. We can see that the peak in the derivative of the free energy corresponds to the drop in the chiral condensate, the latter being a signal of the transition leading to chiral symmetry restoration. The determination of the critical coupling from the peak of the derivative of the free energy is also consistent with 


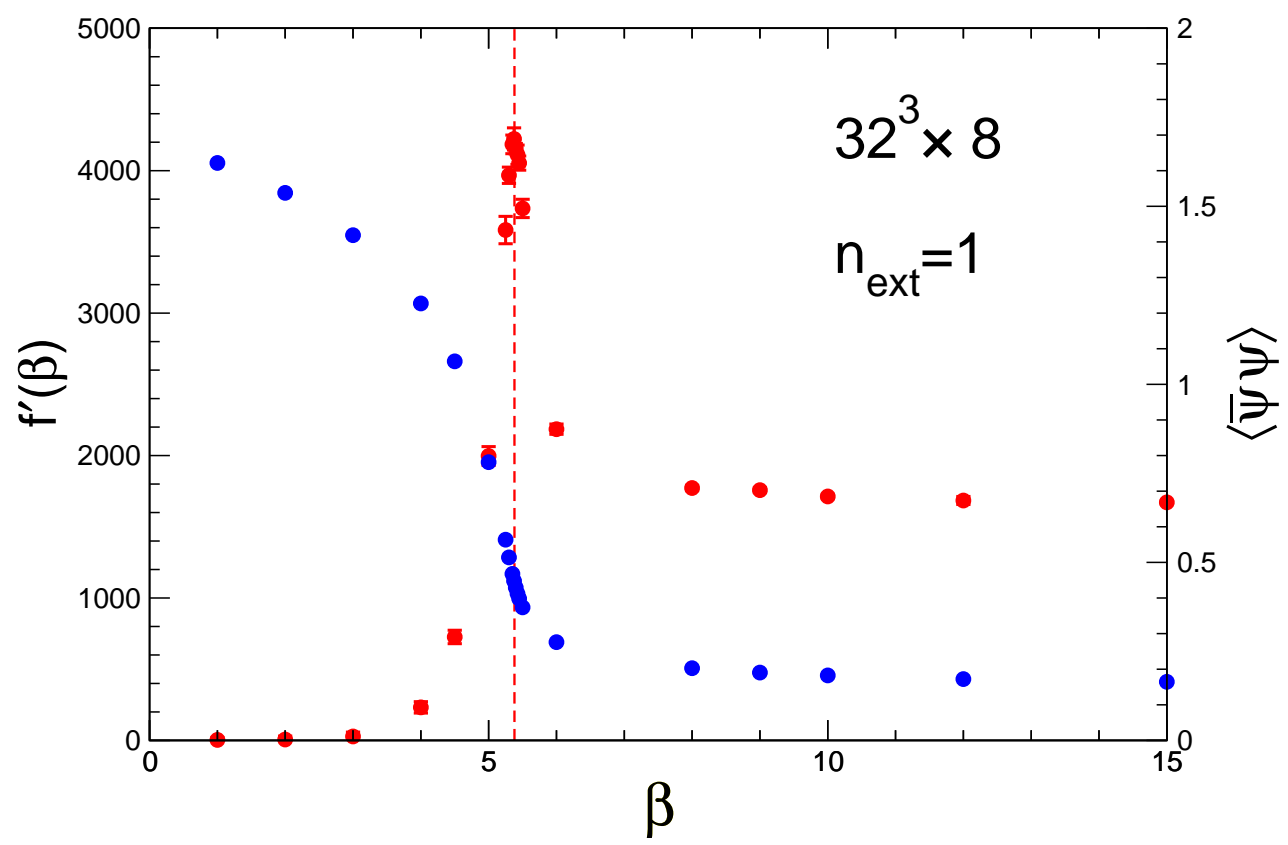

Figure 1: The derivative of the free energy eq. (2.4) with respect to the gauge coupling (left axis, blue circles), and the chiral condensate (right axis, red squares) versus $\beta$. The vertical line represents the position of the peak in the derivative of the free energy.

the determinations obtained by studying the Polyakov and the plaquette susceptibility. Therefore we may conclude that the critical coupling of the phase transition can be located by looking at the peak of the derivative of the free energy and, as in the case of zero external field and within statistical uncertainties, a single transition seems to be present where both deconfinement and chiral symmetry restoration take place.

We have then varied the strength of the external field by tuning up the parameter $n_{\text {ext }}$ and searched for the phase transition signalled by the peak of the derivative of the free energy finding that the critical coupling shifts towards lower values by increasing the external field strength. On the other hand we have found that an abelian monopole background field does not have any influence on the critical coupling. Indeed we have performed numerical simulations in presence of an abelian monopole background field with monopole charge $n_{\text {mon }}=10$ (again for 2 staggered flavors QCD of mass $a m_{q}=0.075$ ). The critical coupling has been estimated to be $\beta_{c}=5.4873(192)$ consistently with the critical coupling $\beta_{c}=5.495(25)$ obtained without any external field.

Once the critical couplings in correspondence of several values of the external abelian chromomagnetic field have been obtained the corresponding critical temperature is given by

$$
T_{c}=\frac{1}{a\left(\beta_{c}, m_{q}\right) L_{t}},
$$

where $L_{t}$ is the lattice temporal size and $a\left(\beta_{c}, m_{q}\right)$ is the lattice spacing at the given critical coupling $\beta_{c}$. We have to face now the problem of fixing the physical scale. In order to reduce the systematic effects involved in this procedure we consider the ratios

$$
\frac{T_{c}(g H)}{T_{c}} \quad \text { vs. } \quad \frac{\sqrt{g H}}{T_{c}}
$$




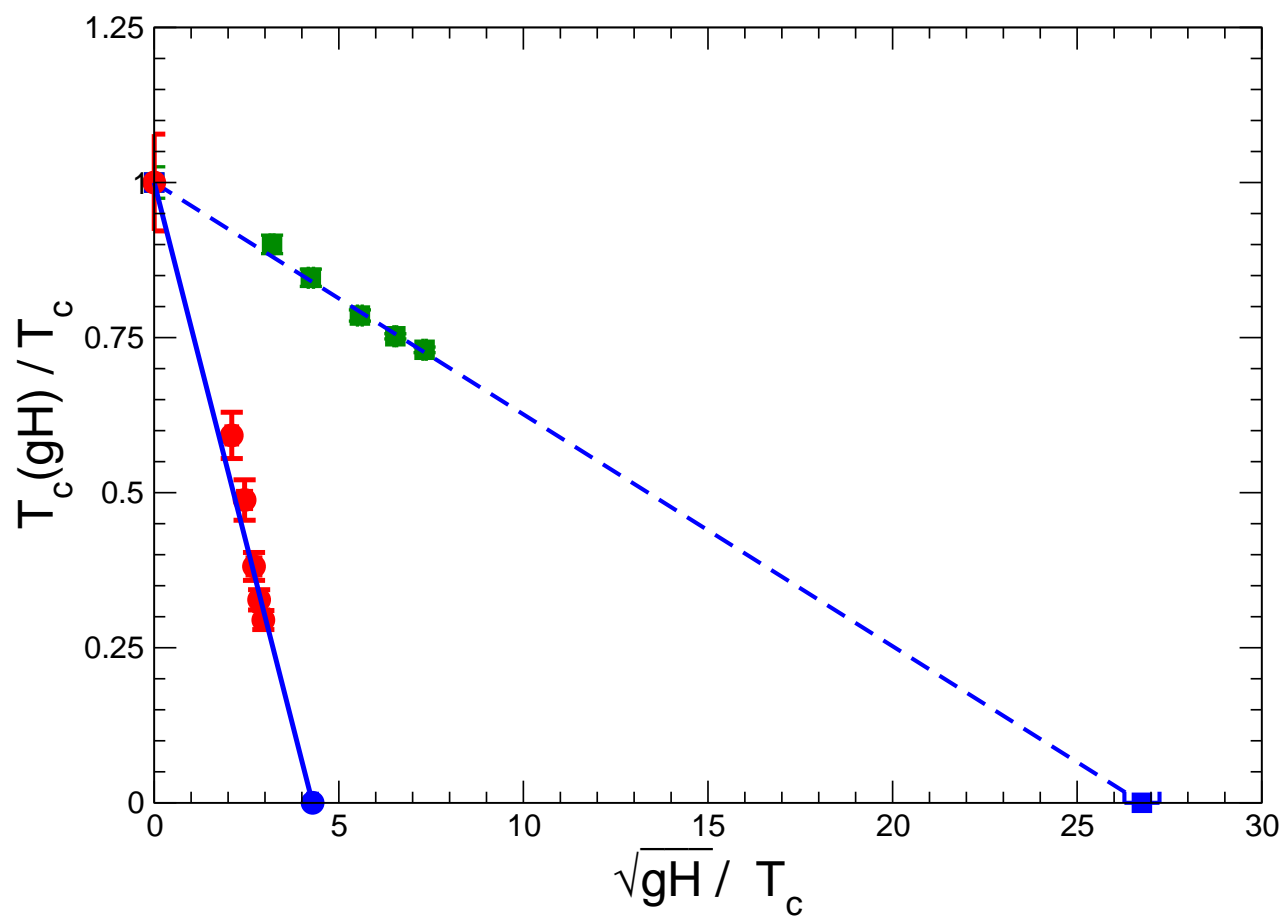

Figure 2: The critical temperature $T_{c}(g H)$ at a given strength of the chromomagnetic background field in units of the critical temperature $T_{c}$ without external field versus the square root of the strength of the background field in the same units. Red circles are obtained by adopting the improved scaling function. The blue line is the linear best fit. The blue circle on the horizontal axis is the linear extrapolated values for the critical background field. Green squares are obtained by adopting the 2-loop scaling function. The blue dashed line is the linear best fit. The blue square on the horizontal axis is the linear extrapolated values for the critical field.

where $T_{c}$ is the critical temperature without external field. The above quantities can be obtained once the ratio of the lattice spacings at the respective couplings is known. A rough estimate of this ratio can be inferred by using the 2-loop scaling function $f\left(g^{2}\right)$

$$
f\left(g^{2}\right)=\left(b_{0} g^{2}\right)^{-b_{1} / 2 b_{0}^{2}} \exp \left(-\frac{1}{2 b_{0} g^{2}}\right)
$$

with

$$
\begin{aligned}
& b_{0}=\frac{1}{16 \pi^{2}}\left[11 \frac{N_{c}}{3}-\frac{2}{3} N_{f}\right] \\
& b_{1}=\left(\frac{1}{16 \pi^{2}}\right)^{2}\left[\frac{34}{3} N_{c}^{2}-\left(\frac{10}{3} N_{c}+\frac{N_{c}^{2}-1}{N_{c}}\right) N_{f}\right] ;
\end{aligned}
$$

$N_{c}$ is the number of colors and $N_{f}$ is the number of flavors. A better estimate could be obtained by exploiting an improved scaling function $f\left(g^{2}\right)\left(1+c_{2} \hat{a}(g)^{2}+c_{4} \hat{a}(g)^{4}\right)$. We do not know, however, the values of $c_{2}$ and $c_{4}$ for $N_{f}=2$. In a first approximation we will fix $c_{2}$ and $c_{4}$ to their quenched values $[10,11]$.

In figure 2 the quantities reported in eq. (3.2) are displayed for both choices described above, i.e. 2-loop asymptotic scaling and improved scaling. Our main result is that, even in presence of 


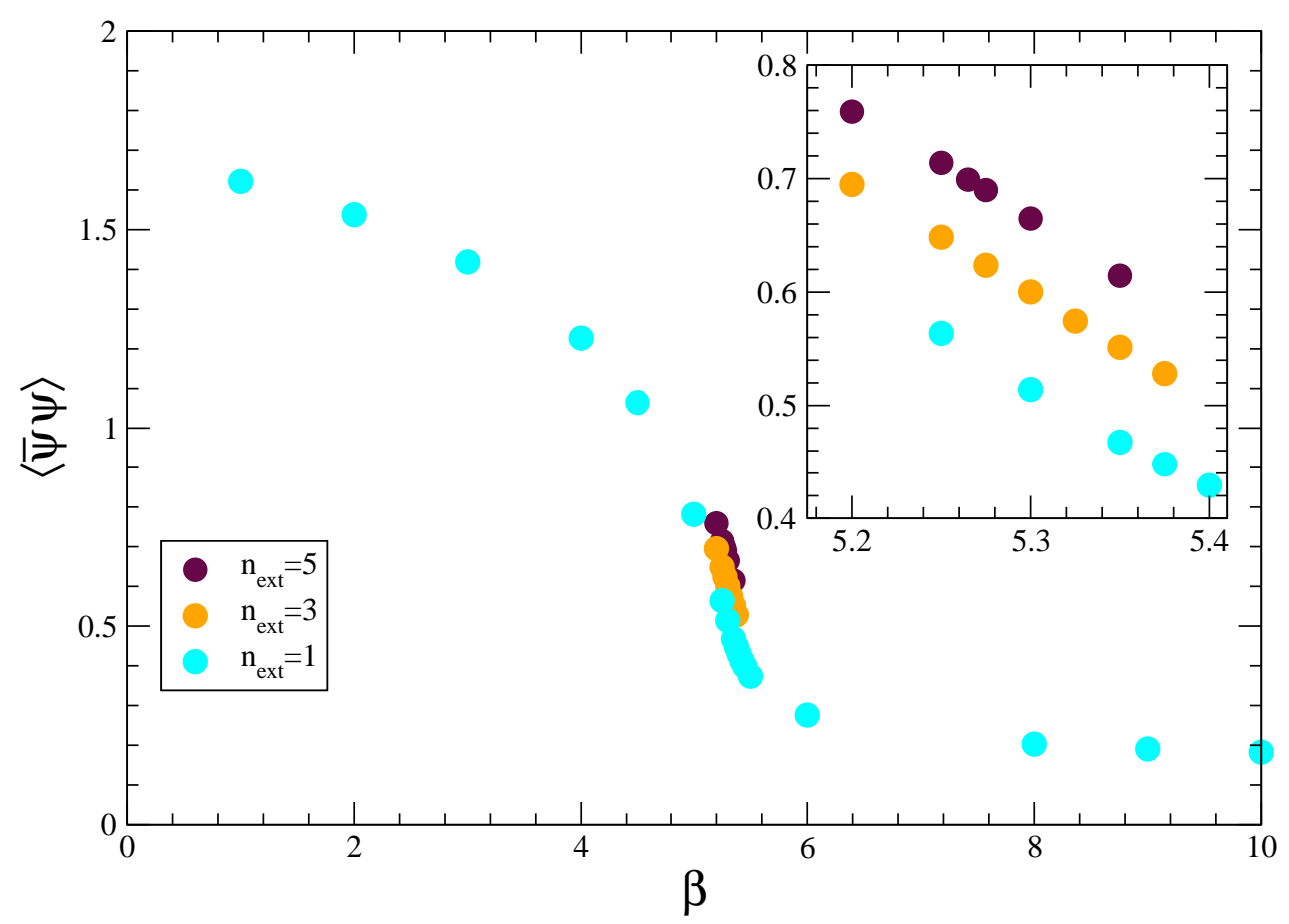

Figure 3: The chiral condensate versus $\beta$ in correspondence of some values of the constant chromomagnetic background field. In the inset the region corresponding to the phase transition has been magnified.

dynamical quarks, the critical temperature decreases with the strength of the chromomagnetic field. Furthermore a linear fit to our data can be extrapolated to very low temperatures leading to the prediction of a critical field strength above which strongly interacting matter should be deconfined at all temperatures. Nevertheless, as can be appreciated from figure 2, the exact value of the critical field strength is largely dependent on the choice of the physical scale: assuming the 2-loop scaling function we obtain $\sqrt{g H_{c}} / T_{c}=26.8(5)$, while using the improved scaling function we obtain $\sqrt{g H_{c}} / T_{c}==4.29(10)$; if the deconfinement temperature at zero field strength is taken to be of the order of $170 \mathrm{MeV}$, that means $\sqrt{g H_{c}}$ in the range $0.7-4.5 \mathrm{GeV}$. Hence in order to get a reliable estimate of the critical field strength requires to get a more reliable estimate of the physical scale of our lattices.

To conclude this section we consider our measurements of the chiral condensate. In figure 3 we display the chiral condensate versus the gauge coupling in correspondence of some values of the external field strength. Numerical data show that, at least in the critical region, the value of the chiral condensate depends on the strength of the applied field. Similar results have been found in ref. [12].

\section{Summary and Conclusions}

We have studied how a constant chromomagnetic field influences the QCD dynamics. By focusing on the finite temperature theory we have found that, analogously to what happens in the pure gauge theory [2], the critical temperature depends on the strength of the constant chromomagnetic background field. More specifically, the critical temperature decreases as the external field 
is increased and, as an extrapolation of our results, for strong enough field strengths the system is always deconfined. A rough estimate of this critical field strength turns out to be of the order of $1 \mathrm{GeV}$, which is a typical QCD scale [13].

What is more, by comparing the critical couplings determined from the derivative of the free energy functional with those determined from the susceptibility of the chiral condensate and of the Polyakov loop we have ascertained that, even in presence of an external chromomagnetic background field and at least up to the field strengths explored in the present work, the critical temperatures where deconfinement and chiral symmetry restoration take place coincide within errors.

Another intriguing aspect that deserves further studies is the dependence of the chiral condensate on the chromomagnetic field strength.

\section{References}

[1] P. Cea and L. Cosmai, Abelian chromomagnetic fields and confinement, JHEP 02 (2003) 031, [hep-lat/0204023].

[2] P. Cea and L. Cosmai, Color dynamics in external fields, JHEP 08 (2005) 079, [hep-lat/0505007].

[3] F. Karsch and M. Lutgemeier, Deconfinement and chiral symmetry restoration in an SU(3) gauge theory with adjoint fermions, Nucl. Phys. B550 (1999) 449-464, [hep-lat/9812023].

[4] J. Engels, S. Holtmann, and T. Schulze, The chiral transition of $N(f)=2 Q C D$ with fundamental and adjoint fermions, PoS LAT2005 (2006) 148, [hep-lat / 0509010 ].

[5] G. Lacagnina, G. Cossu, M. D’Elia, A. Di Giacomo, and C. Pica, Monopole condensation in two-flavour adjoint QCD, hep-lat/0609049.

[6] P. Cea, L. Cosmai, and A. D. Polosa, The lattice Schrödinger functional and the background field effective action, Phys. Lett. B392 (1997) 177-181, [hep-lat/9601010].

[7] P. Cea and L. Cosmai, Probing the non-perturbative dynamics of SU(2) vacuum, Phys. Rev. D60 (1999) 094506, [hep-lat/9903005].

[8] P. Cea, L. Cosmai, and M. D'Elia, The deconfining phase transition in full QCD with two dynamical flavors, JHEP 02 (2004) 018, [hep-lat/ 0401020 ].

[9] S. Gottlieb, W. Liu, D. Toussaint, R. L. Renken, and R. L. Sugar, Hybrid molecular dynamics algorithms for the numerical simulation of quantum chromodynamics, Phys. Rev. D35 (1987) 2531-2542.

[10] C. R. Allton, Lattice Monte Carlo data versus perturbation theory, hep-lat/9610016.

[11] R. G. Edwards, U. M. Heller, and T. R. Klassen, Accurate scale determinations for the Wilson gauge action, Nucl. Phys. B517 (1998) 377-392, [hep-lat/9711003].

[12] J. Alexandre, K. Farakos, S. J. Hands, G. Koutsoumbas, and S. E. Morrison, QED(3) with dynamical fermions in an external magnetic field, Phys. Rev. D64 (2001) 034502, [hep-lat/ 0101011 ].

[13] D. Kabat, K.-M. Lee, and E. Weinberg, QCD vacuum structure in strong magnetic fields, Phys. Rev. D66 (2002) 014004, [hep-ph/ 0204120$].$ 